\title{
Wie schätzen stationäre Patienten mit türkischem Migrationshintergrund die Kultursensibilität in einem deutschen Krankenhaus der Maximalversorgung ein?
}

\author{
Eine Untersuchung zur Religionsausübung, Ernährung und geschlechtsspezifischen \\ Versorgung
}

A. Giese ${ }^{1,2}$, M. Uyar ${ }^{1}$, B. F. Henning ${ }^{1}$, H. H. Uslucan ${ }^{3}$, T. Westhoff ${ }^{1}$, N. Pagonas ${ }^{1}$

Institute

1 Medizinische Klinik I (Innere Medizin), Marienhospital Herne, Universitätsklinikum der Ruhr-Universität Bochum, Herne, Deutschland

2 Medizinische Klinik I, St. Josef-Hospital Bochum, Universitätsklinikum der Ruhr-Universität Bochum, Bochum, Deutschland

3 Stiftung Zentrum für Türkeistudien und Integrationsforschung, Institut an der Universität Duisburg-Essen, Essen, Deutschland

\section{Zusammenfassung}

Hintergrund und Fragestellung | Eine kultursensible Behandlung von Migranten ist ein Ziel im deutschen Gesundheitssystem. Quantitative Daten zum Ist-Zustand aus Sicht der Krankenhauspatienten fehlten bislang.

Patienten und Methoden I Stationäre Patienten mit türkischem Migrationshintergrund ( $\mathrm{T}, \mathrm{n}=121)$ und deutsche Patienten ( $D, n=121)$ eines Krankenhauses der Maximalversorgung wurden in ihrer Muttersprache befragt.

Ergebnisse I 97,5\% der T waren Muslime, 82,6\% der D waren Christen. Mit 88,5\% war der großen Mehrheit der T Religion „wichtig“ oder „sehr wichtig“ (D: 35,8\%). 50,8\% der T hielten die im Krankenhaus gebotenen Möglichkeiten zu beten für „schlecht“ oder „sehr schlecht“ (D: 0,9\%). Für $90 \%$ der T war es „ziemlich“ oder „äußerst“ schwierig, islamische Ernährungsgebote im Krankenhaus einzuhalten. 79,0\% der weiblichen T war gleichgeschlechtliche Pflege „ziemlich“ oder „äu- ßerst" wichtig, bei männlichen T waren es immerhin noch 40,0\% (weibliche D: $36,3 \%$, männliche D: $7,7 \%$ ). Wenn gleichgeschlechtliche Pflege nicht möglich war, gaben $69,7 \%$ der weiblichen $\mathrm{T}$ an, dass für sie die Anwesenheit einer Frau bei Untersuchungen und Behandlungen „ziemlich“ oder „äußerst“ wichtig sei (weibliche D: 25,4\%, männliche T: $28,9 \%$, männliche D: $6,1 \%)$. Eine retrospektive Auswertung ergab, dass 5,8\% aller 8988 im Krankenhaus im Befragungszeitraum aufgenommenen Patienten einen türkischen Migrationshintergrund hatten.

Folgerungen | Um den Bedürfnissen von T gerecht zu werden, sollten Krankenhäuser die Möglichkeit zum Gebet für Muslime verbessern, die Zusammenarbeit mit islamischen Geistlichen anstreben und durch geeignete Kennzeichnungen oder eine Umstellung des Speiseplanes die Einhaltung islamischer Ernährungsgebote vereinfachen. Weibliche, aber auch männliche T wünschten sich vermehrt eine gleichgeschlechtliche pflegerische und ärztliche Betreuung.

\section{Einleitung}

Krankenhäuser in Deutschland sind verpflichtet, ihre Leistungen entsprechend dem jeweiligen Stand der wissenschaftlichen Erkenntnisse und in der fachlich gebotenen Qualität zu erbringen [1]. Hierzu gehört eine angemessene, kultursensible Versorgung der Patienten mit Migrationshintergrund [10, 13, 18]. In der Bundesrepublik Deutschland haben 15,9 Millionen Menschen (19,5\% der Bevölkerung) einen Migrationshintergrund. Mit rund 3 Millionen bilden die türkischen Migranten die größte Gruppe [2]. Die Mehrzahl der türkischen Migranten nimmt bei den Fachkräften des Gesundheitswesens mangelndes Wissen über die eigene Kultur wahr [4]. Eine Studie zur Kultursensibilität in Nordrhein-Westfalen zeigt, dass viele Krankenhäuser keine durchgängige Erfolgskontrolle ihres kultursensiblen Handelns haben und dass nur wenige kultursensible Aspekte umgesetzt werden [5].
Damit für die Verbesserung der Versorgung von Menschen mit Migrationshintergrund eine adäquate Strategie entwickelt werden kann, bedarf es der Identifizierung wichtiger Ansatzpunkte. Untersuchungen zur Sprachkompetenz und zum Verständnis medizinischer Informationen durch Patienten mit türkischem Migrationshintergrund wurden durch uns bereits publiziert [8]. Quantitative Daten zur Einschätzung der Kultursensibilität durch Patienten mit Migrationshintergrund im Krankenhaus fehlen jedoch bislang. Es ist nicht bekannt, wie die Möglichkeit zur Religionsausübung im Krankenhaus eingeschätzt wird und welche Rolle kulturelle Besonderheiten, wie die Einhaltung islamischer Ernährungsgebote oder eine Behandlung durch gleichgeschlechtliches Personal spielen. Wir haben daher stationäre Patienten mit türkischem Migrationshintergrund im Rahmen einer Querschnittstudie zu drei potenziell kultursensiblen Bereichen des Krankenhausalltages (Religionsausübung, Ernährung, geschlechtsspezifische Behandlung) befragt. 


\section{Methode}

Es handelt sich bei der Studie um eine Teilauswertung von Daten, die im Rahmen einer umfassenden Untersuchung von stationären Patienten mit türkischem Migrationshintergrund in Deutschland, erhoben wurden. Konsekutiv rekrutierte Patienten mit türkischem Migrationshintergrund $(\mathrm{T})$ und deutsche Patienten ohne Migrationshintergrund (D) wurden wie zuvor beschrieben [8] prospektiv in die Studie eingeschlossen. Die Studienteilnehmer beantworteten einen Fragebogen in der jeweiligen Muttersprache (T: türkisch, D: deutsch). Die notwendige Größe des Studienkollektivs wurde vor Studienbeginn durch eine Fallzahlberechnung abgeschätzt [11]. Wir haben eine Varianz (Sigma) von 1,2 in beiden Gruppen, eine Effektstärke von 0,5 sowie eine Fehlerwahrscheinlichkeit (Alpha) von $5 \%$ angenommen. Daraus ergab sich, dass mindestens 92 Patienten in jeder Gruppe notwendig sind, um eine Power von über $80 \%$ zu erreichen. Vor diesem Hintergrund haben wir eine Teilnehmerzahl von 121 Patienten in jeder Studiengruppe festgelegt. Die Rekrutierung wurde beendet, sobald dieses Ziel erreicht war.

Studienpopulation I Die Studie wurde am Marienhospital Herne, das sich in katholischer Trägerschaft befindet, durchgeführt. Dieses Krankenhaus der Maximalversorgung ist Universitätsklinikum der Ruhr-Universität Bochum und versorgte 2001 über 20000 stationäre Aufnahmen (575 Planbetten). Die Studie erfolgte an dem größeren der beiden Standorte mit 362 Planbetten.

Einschlusskriterien der Befragung | Patienten im Alter von mindestens 18 Jahren wurden zwischen dem zweiten und dritten Tag ihres Krankenhausaufenthaltes prospektiv in die Studie eingeschlossen. Patienten mit türkischem Migrationshintergrund wurden anhand ihres Namens unter den stationären Patienten identifiziert [16]. Vor Durchführung der Befragung wurde sichergestellt, dass ein türkischer Migrationshintergrund im Sinne der Definition des Mikrozensus 2005 [7] gegeben war. Deutsche Patienten ohne Migrationshintergrund dienten als Kontrollen (D).

Entwicklung des Fragebogens und Durchführung der Befragung | Für die von uns untersuchten Bereiche: Religionsausübung, kulturspezifische Ernährung, geschlechtsspezifische Pflege/Behandlung, existiert bislang kein Fragenkatalog. Wir entwickelten daher einen Fragebogen und graduierten die Antworten im Sinne der Likert-Skala, um eine Quantifizierbarkeit und Vergleichbarkeit der Befragungsergebnisse sicherzustellen (z. B. 1: sehr schlecht, 2: schlecht, 3: mittelmäßig, 4: gut, 5: sehr gut). Die Fragen sind als zusätzliches Material zu dem Artikel online (https://www.thieme-connect. com/products/ejournals/abstract/10.1055/ s-0041-100007) abrufbar. Aus ethischen Gründen durfte die Befragung der Patienten nicht mit notwendigen Untersuchungen, Therapien und Ruhezeiten kollidieren und die Patienten sollten nicht in ihren Aktivitäten (Besuch etc.) eingeschränkt werden. Die Befragung fand am zweiten oder dritten Tag des stationären Aufenthaltes statt. Alle Studienteilnehmer stimmten dem Studieneinschluss nach Aufklärung zu. Für die Durchführung der Studie liegt das Einverständnis der Ethikkommission der Medizinischen Fakultät der Ruhr-Universität Bochum vor.

Untersuchung zu den nichtbefragten Patienten im Studienzeitraum | Unter den Patienten, die im Befragungszeitraum (30.05.2011-29.02.2012) im Krankenhaus stationär aufgenommen wurden, wurde der türkische Migrationshintergrund retrospektiv ermittelt. Dazu wurde ein onomastischer Algorithmus [16] verwendet, ein Verfahren mit dem türkische Namen in der Datenbank identifiziert werden können.

Auswertung I Die statistische Auswertung wurde mit SPSS (Version 20.0.0, Chicago, USA) durchgeführt. Kontinuierliche Daten wurden mit dem Student-t-Test verglichen. Einige der Fragen hatten graduierte Antwortmöglichkeiten, denen Zahlenwerte zugeordnet wurden, die wie kontinuierliche Daten gehandhabt wurden. Kategoriale Daten wurden mit dem exakten Test nach Fischer verglichen.

\section{Ergebnisse}

- Tab. 1 zeigt wichtige Charakteristika der befragten Patienten. Die große Mehrheit der T war mus-

Tab. 1 Charakteristika der befragten stationären Patienten

Tab. 1 Charakteristika der befragten stationären Patienten

$\begin{array}{lll}\text { Deutsche* (D) } & \text { P } & \text { Türkischer } \\ & \text { Migrations- }\end{array}$

hintergrund ( $T$ )

Demografische Merkmale

n

121

Alter in J, MW $\pm \mathrm{SA}$

$56,9 \pm 16,8$

$<0,001$

$44,9 \pm 17,8$

Männer n (\% der Gruppe)

$66(54,5)$

0,010

$45(37,2)$

Nationalität

Deutsch

$121(100)<0,001$

$37(30,5)$

Türkisch

$0(0)$

$<0,001$

$80(66,1)$

Deutsch und türkisch

$0(0)$

0,122

$4(3,3)$

Religion

Christentum

$100(82,6)$

$<0,001$

$0(0)$

Islam

$0(0)$

$<0,001$

$118(97,5)$

Sonstige

$1(0,8)$

1,0

$1(0,8)$

Keine

$19(15,7)$

$<0,001$

$2(1,7)$

Fehlende Angabe

$1(0,8)$

1,0

$0(0)$

* oder D: befragte deutsche Patienten ohne Migrationshintergrund. T: befragte Patienten mit türkischem Migrationshintergrund. p: Signifikanzwert. n: Anzahl. MW: Mittelwert. SA: Standardabweichung. Werte in Klammern: Prozent der Gruppe 
Tab. 2 Religionsausübung im Krankenhaus limisch (97,5\%) während die Mehrheit der D christlich war (82,6\%).

Die Ergebnisse der Befragung zur Religionsausübung im Krankenhaus sind in $>$ Tab. 2 zusammengefasst. T und D unterschieden sich bezüglich ihrer Religiosität und ihrer Einschätzung der Möglichkeiten zur Religionsausübung im Krankenhaus. 88,8\% der T gaben an, dass ihnen Religion im Leben „ziemlich“ oder „äußerst“ wichtig sei (D: 38,5\%). 50,9\% der T hatten das Gefühl, dass Pflegekräfte und Ärzte ihre Religion „ziemlich“ oder „äußerst“" ernst nahmen (D: 26,9\%). Nur etwas mehr als $10 \%$ der T fühlten sich in ihrer Religion „überhaupt nicht“ oder „wenig“ ernst genommen. Die Möglichkeit zum Gebet schätzten 50,8\% der T als „sehr schlecht“ oder „schlecht“ ein, was im starken Gegensatz zu der Mehrheit der Deutschen stand, die ihre Möglichkeit zum Gebet mehrheitlich (90,5\%) „gut“ oder „sehr gut“ fanden. Unter den T wäre es 49,6\% der Befragten „ziemlich“ oder „äußerst“ wichtig gewesen, im Krankenhaus einen religiösen Ansprechpartner zu haben (D: 11,6\%).

Ein Teil der Fragen zielte auf die Bedeutung der pflegerischen oder ärztlichen Behandlung durch gleichgeschlechtliches Personal ab. Auch die Wichtigkeit der Anwesenheit einer gleichgeschlechtlichen dritten Person bei Untersuchungen und Behandlungen durch andersgeschlechtliches Personal wurde bewertet. Wir haben die Antworten hierzu in $>$ Tab. 3 nach Geschlecht getrennt dargestellt. T war es demnach wichtiger als D, von einer gleichgeschlechtlichen Pflegekraft oder von einer/einem gleichgeschlechtlichen Ärztin/Arzt behandelt zu werden und bei Unter- suchungen eine gleichgeschlechtliche dritte Person anwesend zu wissen. Dies galt insbesondere für die Frauen unter den T. 79\% der weiblichen T war es „ziemlich“ oder „äußerst“ wichtig, von einer Frau gepflegt zu werden (weibliche D: 36,6\%). Jedoch wünschten sich auch viele männliche $\mathrm{T}$ eine geschlechtsspezifische Behandlung. Der Anteil der männlichen $\mathrm{T}$, denen eine geschlechtsspezifische Behandlung „ziemlich“ oder „äußerst“ wichtig war, überstieg in allen abgefragten Bereichen den Anteil der deutschen Frauen.

Die Ergebnisse der Befragung zum Thema Religion und Essen sind in $>$ Tab. 4 zusammengefasst. $\mathrm{Zu}$ diesem Thema wurden nur T befragt. 90\% der T gaben an, die Einhaltung islamischer Gebote beim Essen sei für sie im Krankenhaus „ziemlich“ oder „äußerst“ schwierig gewesen. Die Einhaltung dieser Vorschriften war 30,6\% der T „wichtig“ oder „sehr wichtig“. 17,3\% fühlten sich „ziemlich“ oder „äußerst“ stark darauf angewiesen, dass ihnen ihre Familie Essen mitbringt, um sich im Krankenhaus gut ernähren zu können.

Von den $121 \mathrm{~T}$ gehörten 53 der ersten, 49 der zweiten, 18 der dritten und einer der vierten Einwanderergeneration an. Die Einwanderergenerationen unterschieden sich hinsichtlich ihres Alters, der in Deutschland verbrachten Lebenszeit und in ihrer selbst-eingeschätzten Sprachkompetenz. Tab. 5 zeigt, dass hinsichtlich des Stellenwertes der Religion, des Wunsches nach einem religiösen Beistand im Krankenhaus und bezüglich der Einhaltung islamischer Essensvorschriften keine signifikanten Unterschiede zwischen Einwanderern der 1., 2. und 3. bis 4 . Generation be-

\begin{tabular}{|c|c|c|c|c|c|c|c|}
\hline $\begin{array}{l}\text { Anzahl } \\
\text { Teilnehmer }\end{array}$ & $\begin{array}{l}1 \\
\text { überhaupt nicht/ } \\
\text { sehr schlecht* }\end{array}$ & $\begin{array}{l}2 \\
\text { wenig/ } \\
\text { schlecht* }\end{array}$ & $\begin{array}{l}3 \\
\text { mittelmäßig }\end{array}$ & $\begin{array}{l}4 \\
\text { ziemlich / } \\
\text { gut* }^{*}\end{array}$ & $\begin{array}{l}5 \\
\text { äußerst / } \\
\text { sehr gut* }\end{array}$ & $\mathrm{MW} \pm \mathrm{SA}$ & $\mathbf{p}$ \\
\hline \multicolumn{8}{|c|}{ Stellenwert der Religion „Wie wichtig ist Ihnen Religion in Ihrem Leben?“ } \\
\hline$T(n=121) n(\%)$ & $1(0,8)$ & $2(1,7)$ & $11(9,1)$ & $21(17,4)$ & $86(71,1)$ & $4,6 \pm 0,8$ & \multirow{2}{*}{$p<0,001$} \\
\hline$D(n=120) n(\%)$ & $15(12,5)$ & $17(14,2)$ & $45(37,5)$ & $31(25,8)$ & $12(10)$ & $3,1 \pm 1,1$ & \\
\hline
\end{tabular}

Aufmerksamkeit der Ärzte bezüglich der Religion „Wie stark nehmen die Ärzte im Krankenhaus Ihre Religion ernst?“

$\begin{array}{llllllll}\mathbf{T}(\mathbf{n}=\mathbf{1 1 4}) \mathbf{n}(\%) & 6(5,3) & 6(5,3) & 44(38,6) & 44(38,6) & 14(12,3) & 3,5 \pm 1,0 & \mathbf{0}<0,001 \\ \mathbf{D}(\mathbf{n = 9 3 )} \mathbf{n}(\%) & 18(19,4) & 15(16,1) & 35(37,6) & 15(16,1) & 10(10,8) & 2,8 \pm 1,2\end{array}$

Aufmerksamkeit der Pflege bezüglich der Religion „Wie stark nehmen die Schwestern/Pfleger im Krankenhaus Ihre Religion ernst?“

\begin{tabular}{|c|c|c|c|c|c|c|c|}
\hline$T(n=114) n(\%)$ & $3(2,6)$ & $9(7,9)$ & $44(38,6)$ & $45(39,5)$ & $13(11,4)$ & $3,5 \pm 0,9$ & \multirow{2}{*}{$\mathrm{p}<0,001$} \\
\hline$D(n=93) n(\%)$ & $18(19,4)$ & $15(16,1)$ & $35(37,6)$ & $17(18,3)$ & $8(8,6)$ & $2,8 \pm 1,2$ & \\
\hline \multicolumn{8}{|c|}{ Möglichkeit zum Gebet „Wie gut ist im Marienhospital Ihre Möglichkeit zu beten?“** } \\
\hline$T(n=120) n(\%)$ & $37(30,8)$ & $24(20,0)$ & $39(32,5)$ & $14(11,7)$ & $6(5)$ & $2,4 \pm 1,2$ & \multirow{2}{*}{$\mathrm{p}<0,001$} \\
\hline$D(n=115) n(\%)$ & $0(0)$ & $1(0,9)$ & $10(8,7)$ & $83(72,2)$ & $21(18,3)$ & $4,1 \pm 0,5$ & \\
\hline
\end{tabular}

Religiöser Beistand „Wie wichtig ist es Ihnen, im Krankenhaus einen religiösen Ansprechpartner (Priester/Imam) zu haben, der Ihnen bei Fragen im Umgang mit religiösen Geboten während der Krankheit helfen kann?“

$\mathbf{T}(\mathbf{n}=\mathbf{1 2 1}) \mathbf{n}(\%) \quad 31(25,6)$

$\begin{array}{lll}11(9,1) & 19(15,7) & 22(18,2) \\ 37(30,8) & 22(18,3) & 10(8,3)\end{array}$

$38(31,4)$

$3,2 \pm 1,6$

$2,1 \pm 1,1$

T: befragte Patienten mit türkischem Migrationshintergrund. D: befragte Patienten ohne Migrationshintergrund. n: Anzahl. MW: Mittel der Werte nach Likert-Skala. SA: Standardabweichung. p: Signifikanz. Werte in Klammern: Prozent der Gruppe 


$\begin{array}{llllllll}\text { Anzahl } & 1 & 2 & 3 & 4 & 5 & \\ \text { Teilnehmer } & \begin{array}{l}\text { überhaupt } \\ \text { nicht }\end{array} & \text { wenig } & \text { mittelmäßg } & \text { ziemlich } & \text { äußerst } & \text { MW } \pm S A & \text { P }\end{array}$

Gleichgeschlechtliche Pflege „Wie wichtig ist es Ihnen, von einer Pflegekraft Ihres Geschlechts gepflegt zu werden?“

$\begin{array}{lllllllll}\text { Frauen } & T(n=76) n(\%) & 7(9,2) & 2(2,6) & 7(9,2) & 17(22,4) & 43(56,6) & 4,1 \pm 1,3 & p<0,001 \\ & D(n=55) n(\%) & 17(30,9) & 6(10,9) & 12(21,8) & 7(12,7) & 13(23,6) & 2,8 \pm 1,6 & \\ \text { Männer } & T(n=45) n(\%) & 21(46,7) & 2(4,4) & 4(8,9) & 9(20,0) & 9(20,0) & 2,6 \pm 1,7 & p<0,001 \\ & D(n=65) n(\%) & 46(70,8) & 10(15,4) & 4(6,2) & 4(6,2) & 1(1,5) & 1,5 \pm 1,0 \quad P\end{array}$

Gleichgeschlechtliche Ärztin/Arzt „Wie wichtig ist es Ihnen, von einer Ärztin/einem Arzt Ihres Geschlechts untersucht und behandelt zu werden?"

\begin{tabular}{|c|c|c|c|c|c|c|c|c|}
\hline \multirow[t]{2}{*}{ Frauen } & $T(n=76) n(\%)$ & $13(17,1)$ & $5(6,6)$ & $13(17,1)$ & $17(22,4)$ & $28(36,8)$ & $3,6 \pm 1,5$ & \multirow{2}{*}{$\mathrm{p}<0,001$} \\
\hline & $D(n=55) n(\%)$ & $25(45,5)$ & $11(20,0)$ & $12(21,8)$ & $4(7,3)$ & $3(5,5)$ & $2,1 \pm 1,2$ & \\
\hline \multirow[t]{2}{*}{ Männer } & $T(n=45) n(\%)$ & $23(51,1)$ & $1(2,2)$ & $5(11,1)$ & $5(11,1)$ & $11(24,4)$ & $2,5 \pm 1,7$ & \multirow{2}{*}{$p<0,001$} \\
\hline & $D(n=65) n(\%)$ & $52(80,0)$ & $9(13,8)$ & $3(4,6)$ & $0(0)$ & $1(1,5)$ & $1,3 \pm 0,7$ & \\
\hline
\end{tabular}

Gleichgeschlechtliche dritte Person „Wie wichtig ist es Ihnen, dass bei Untersuchungen und Behandlungen durch einen Mann/eine Frau (gegengeschlechtlich) immer eine gleichgeschlechtliche Person zugegen ist?“

$\begin{array}{lllllllll}\text { Frauen } & T(n=76) n(\%) & 12(15,8) & 3(3,3) & 8(10,5) & 25(32,9) & 28(36,8) & 3,7 \pm 1,4 & p<0,001 \\ & D(n=55) n(\%) & 27(49,1) & 7(12,7) & 7(12,7) & 8(14,5) & 6(10,9) & 2,3 \pm 1,5 & \\ \text { Männer } & T(n=45) n(\%) & 20(44,4) & 5(11,1) & 7(15,6) & 6(13,3) & 7(15,6) & 2,4 \pm 1,5 \\ & D(n=65) n(\%) & 49(75,4) & 11(16,9) & 1(1,5) & 3(4,6) & 1(1,5) & 1,4 \pm 0,9\end{array}$

T: befragte Patienten mit türkischem Migrationshintergrund. D: befragte deutsche Patienten ohne Migrationshintergrund. n: Anzahl. MW: Mittel der Werte nach Likert-Skala. SA: Standardabweichung. p: Signifikanz. Werte in Klammern: Prozent der Gruppe

Tab. 3 Geschlechtsspezifische Behandlung

standen. Lediglich der Wunsch nach einer gleichgeschlechtlichen Pflegekraft war bei der 1. Generation ausgeprägter als bei der 3 . und 4 .

In den 9 Monaten des Referenzzeitraumes gab es insgesamt 8988 stationäre Aufnahmen in den betreffenden Standort des Marienhospitals Herne. 5,8\% dieser Patienten hatte einen türkischen Migrationshintergrund.

\section{Diskussion}

Religionsausübung I Der Anteil der Muslime unter den $\mathrm{T}$ in unserer Studie $(97,5 \%)$ war ver- gleichbar mit dem Ergebnis einer repräsentativen Umfrage unter türkischstämmigen Migranten in NRW (Muslim-Anteil: 94,8\%) [15]. In einer anderen Studie gaben türkische oder kurdische ambulante Patienten auf einer Skala von 1 bis 5 (Extreme: „gar nicht“ bis „sehr“) an, wie wichtig ihnen Religion ist [17]. Die Ergebnisse (Migranten: 4,0 $\pm 1,2$, Deutsche: $2,7 \pm 1,3, p=0,001)$ sind mit unseren, an stationären Patienten erhobenen, Werten von der Tendenz her vergleichbar. Erfreulich ist aus unserer Sicht die Tatsache, dass T sich mehrheitlich durch Ärzte und Pflegepersonal in ihrer Religion ernst genommen fühlten. Korrespondierend hierzu sieht die Mehrzahl $(77,2 \%)$ der Krankenhäuser in NRW die Notwen-

Tab. 4 Religion und Essen

$\begin{array}{lllllll}\text { Anzahl Teilnehmer } & \mathbf{1} & \mathbf{2} & \mathbf{3} & \mathbf{4} & \mathbf{5} \\ & \text { überhaupt nicht } & \begin{array}{l}\text { wenig/ } \\ \text { eher nicht* }\end{array} & \begin{array}{l}\text { mittelmäßig/ } \\ \text { halbwegs* }\end{array} & \begin{array}{l}\text { ziemlich/ } \\ \text { überwiegend* }\end{array} & \begin{array}{l}\text { äußerst/ } \\ \text { völlig* }\end{array}\end{array}$

Stellenwert islamischer Ernährungsgebote „Wie wichtig ist Ihnen beim Essen die Einhaltung islamischer Gebote (z.B. Verzicht auf Alkohol, Schweinefleischverbot, Halal-Schlachtung)?“* *

$\mathbf{T}(\mathbf{n = 1 2 1 )} \mathbf{n}$ (\%) $\quad 35(28,9)$

$30(24,8)$

$27(22,3)$

$10(8,3)$

$2,7 \pm 1,3$

Möglichkeit zur Einhaltung islamischer Ernährungsgebote „Wie schwierig ist es für Sie, im Krankenhaus islamische Gebote beim Essen einzuhalten?"“

$T(n=121) \mathbf{n}(\%)$

$3(2,5)$

$2(1,7)$

$7(5,8)$

$13(10,7)$

$96(79,3)$

$4,6 \pm 0,9$

Stellenwert mitgebrachter Nahrung „Wie stark sind Sie im Krankenhaus darauf angewiesen, dass Ihnen Ihre Familie Essen mitbringt, damit Sie sich gut ernähren können?“

$\mathbf{T}(\mathbf{n = 1 2 1 )} \mathbf{n}(\%) \quad 45(37,2) \quad 25(20,7) \quad 30(24,8) \quad 16(13,2) \quad 5(4,1) \quad 2,3 \pm 1,2$

T: befragte Patienten mit türkischem Migrationshintergrund. n: Anzahl. MW: Mittel der Werte nach Likert-Skala. SA: Standardabweichung. p: Signifikanz. Werte in Klammern: Prozent der Gruppe 
digkeit der Berücksichtigung religiöser Vorstellungen bei Migranten [5]. Dass die mehrheitlich muslimischen T schlechtere Möglichkeiten zum Beten sahen als die mehrheitlich christlichen D, erstaunt aufgrund der christlich-katholischen Ausrichtung unseres Krankenhauses nicht. Die Einrichtung eines Gebetsraumes für Muslime oder eines neutral gestalteten Religionsraumes (über letzteres verfügen 36,8\% der Krankenhäuser in NRW [5]) zusätzlich zu der vorhandenen Kapelle sollte aufgrund der vorliegenden Untersuchung erwogen werden. Aus unserer Sicht wäre es für viele Krankenhäuser angemessen, analog zu den christlichen Seelsorgern ein entsprechendes muslimisches Angebot zu schaffen. Neben seelsorgerischen Tätigkeiten ist der Umgang mit religiösen Pflichten (z.B. Fastengebot) einer der Bereiche, in denen ein islamischer Geistlicher einen stationären Krankenhausaufenthalt begleiten kann [9]. Zusätzlich oder alternativ könnten auch interkulturelle Mediatoren sinnvoll sein [14].

Geschlechtsspezifische Behandlung | $87 \%$ der Krankenhäuser in NRW sehen die Notwendigkeit einer verstärkten Berücksichtigung kulturspezifischer Unterschiede des Schamgefühls von Migranten [5]. Die vorliegenden Daten belegen, dass auch bei Männern mit türkischem Migrationshintergrund auf eine Berücksichtigung des Schamgefühls geachtet werden sollte. Die Ergebnisse der Subgruppenanalyse in $\downarrow$ Tab. 5 zeigen, dass jüngere Patienten der 3. und 4. Einwanderergeneration weniger Wert auf eine gleichgeschlechtliche Pflege legten als ältere T der ersten Einwanderergeneration.
Ernährung | Die Ergebnisse unserer Befragung zeigen Defizite bei der Speiseversorgung von T. Passend dazu ist der Anteil der Krankenhäuser in NRW, die nach religiösen Vorschriften zubereitete Gerichte anbieten, mit 22,8\% niedrig [5]. $68,4 \%$ der Krankenhäuser geben an, ob Gerichte mit Schweinefleisch oder -fett zubereitet sind. Unter den Menschen mit türkischem Migrationshintergrund steigt der Anteil der Religiösen $[12,15]$, somit könnte eine mit religiösen Vorschriften kompatible Krankenhausernährung (sowie andere auf die Religion abzustimmende Aspekte des Krankenhausaufenthaltes) in $\mathrm{Zu}$ kunft noch wichtiger werden. Eine verständliche Kennzeichnung der Nahrungsbestandteile auf dem Essensplan sollte ohne finanziellen Mehraufwand in allen Kliniken möglich sein und Patienten mit türkischem Migrationshintergrund die Ernährung im Krankenhaus erleichtern.

Charakteristika der nicht befragten Patienten im Studienzeitraum I In dem Befragungszeitraum hatten 5,8\% der stationär aufgenommenen Patienten einen türkischen Migrationshintergrund. Dies entspricht etwa dem, anhand bundes- [2] und landesweit [3] erhobener Daten, geschätzten Anteil dieser Minderheit in der lokalen Bevölkerung. Bundesweit liegt das Alter der Menschen mit türkischem Migrationshintergrund bei durchschnittlich 31,5 Jahren (ohne Migrationshintergrund: 46,1 Jahre) [2]. Passend dazu ergibt sich zwischen den stationären Aufnahmen mit (45,6 $\pm 18,4$ Jahre) und ohne $(63,6 \pm 16,8$ Jahre $)$ türkischen Migrationshintergrund ein ähnlicher Altersunterschied. Das untersuchte Kranken-

Tab. 5 Charakteristika der befragten Patienten mit türkischem Migrationshintergrund nach Einwanderergeneration (MW $\pm S A$ ).

\begin{tabular}{|c|c|c|c|c|c|c|}
\hline Merkmal & $\begin{array}{l}\text { 1. Gen. } \\
(n=53)\end{array}$ & $\begin{array}{l}\text { p } \\
\text { 1. vs. 2. Gen. }\end{array}$ & $\begin{array}{l}\text { 2. Gen. } \\
(n=49)\end{array}$ & $\begin{array}{l}\text { P } \\
\text { 2. vs 3.+4. Gen. }\end{array}$ & $\begin{array}{l}\text { 3. + 4. Gen. } \\
(n=19)\end{array}$ & $\begin{array}{l}\text { p } \\
\text { 1. vs. 3.+4. Gen. }\end{array}$ \\
\hline Alter in Jahren & $58,1 \pm 15,8$ & $<0,0001$ & $38,9 \pm 10,6$ & $<0,0001$ & $24,0 \pm 4,0$ & $<0,0001$ \\
\hline In Deutschland verlebte Jahre & $29,6 \pm 12,9$ & 0,81 & $30,1 \pm 8,7$ & $<0,0001$ & $24,0 \pm 4,0$ & 0,006 \\
\hline $\begin{array}{l}\text { Wie schätzen Sie Ihre deutschen } \\
\text { Sprachkenntnisse ein?* } \\
\text { ( } 1 \text { = sehr schlecht bis } 5=\text { sehr gut) }\end{array}$ & $2,38 \pm 0,84$ & $<0,0001$ & $3,90 \pm 0,94$ & 0,009 & $4,53 \pm 0,61$ & $<0,0001$ \\
\hline $\begin{array}{l}\text { Wie wichtig ist lhnen lhre Religion?* } \\
\text { (1= überhaupt nicht, } 5=\text { äußerst) }\end{array}$ & $4,72 \pm 0,60$ & 0,051 & $4,43 \pm 0,84$ & 0,855 & $4,47 \pm 1,07$ & 0,230 \\
\hline $\begin{array}{l}\text { Wie wichtig ist es Ihnen, im } \\
\text { Krankenhaus einen religiösen } \\
\text { Ansprechpartner zu haben?* } \\
\text { (1= überhaupt nicht bis } 5=\text { äußerst) }\end{array}$ & $3,30 \pm 1,58$ & 0,856 & $3,24 \pm 1,59$ & 0,359 & $2,84 \pm 1,68$ & 0,287 \\
\hline $\begin{array}{l}\text { Wie wichtig ist lhnen eine gleichge- } \\
\text { schlechtliche Pflegekraft?* } \\
\text { ( } 1 \text { = überhaupt nicht bis } 5=\text { äußerst) }\end{array}$ & $3,81 \pm 1,50$ & 0,440 & $3,57 \pm 1,62$ & 0,168 & $2,95 \pm 1,75$ & 0,044 \\
\hline $\begin{array}{l}\text { Wie wichtig ist lhnen beim Essen die } \\
\text { Einhaltung islamischer Gebote? }{ }^{*} \\
\text { ( } 1 \text { = überhaupt nicht bis } 5=\text { äußerst) }\end{array}$ & $2,60 \pm 1,42$ & 0,844 & $2,55 \pm 1,28$ & 0,142 & $3,05 \pm 1,18$ & 0,221 \\
\hline
\end{tabular}


haus ist konfessionell (katholisch), was die Zusammensetzung der $\mathrm{T}$ und der $\mathrm{D}$ beeinflusst haben könnte. Die Stichprobe der deutschen Patienten ohne Migrationshintergrund (D) war im Vergleich zu den übrigen, nicht befragten deutschen Patienten etwas jünger $(56,9 \pm 16,8$ vs. $63,7 \pm 16,8, p<0,001)$. Aus dem Studiendesign mit einer zuvor festgelegten Teilnehmerzahl in beiden Gruppen ergibt sich zudem, dass 23,2\% der im Krankenhaus aufgenommenen T aber nur $1,4 \%$ der D an der Befragung teilnahmen. Hierdurch ist das Kollektiv der D weniger repräsentativ abgebildet als das der T. Es ist denkbar, dass alle genannten Faktoren zu einem Bias bei der Patientenauswahl unter den Befragungsteilnehmern geführt haben.

Die vorliegende Studie ist die erste, die Krankenhauspatienten beider Geschlechter zu den Themenkomplexen Religionsausübung, kulturspezifische Ernährung, geschlechtsspezifische Pflege und Behandlung befragt hat. Bisher existierten lediglich Daten von türkischen Migrantinnen [6]. Unsere Untersuchung zeigt wichtige Tendenzen unter den befragten Gruppen auf und bereitet den Boden für weitere Arbeiten zu diesem relevanten Themengebiet. Zukünftige Untersuchungen könnten zur Verbesserung der Repräsentativität und Generalisierbarkeit multizentrisch durchgeführt werden. Dies würde ermöglichen, unterschiedliche Krankenhaustypen abzubilden, eine größere Studienpopulation einzuschließen und eine differenziertere Auswertung (z.B. nach Alter, Art der Erkrankung und bei Migranten nach dem Grad der Integration und der Sprachkompetenz) vorzunehmen. Auch weitere Kriterien der Kultursensibilität könnten mit aufgenommen werden. Ebenso sollte der Einschluss weiterer Minderheiten erwogen werden. Sinnvoll wäre zudem eine synchrone Erhebung der tatsächlichen ärztlichen und pflegerischen Betreuung von D und T, um den Ist-Zustand und den Optimierungsbedarf noch präziser darzustellen.

\section{Literatur}

1 SGB V. §135a

2 Bevölkerung und Erwerbstätigkeit - Bevölkerung mit Migrationshintergrund, Ergebnisse des Mikrozensus 2011. In. Wiesbaden: Statistisches Bundesamt; 2012: 1-382

3 Bevölkerung nach Migrationsstatus regional Ergebnisse des Mikrozensus 2010. In. Wiesbaden: Statistische Ämter des Bundes und der Länder; 2013: 1-423

4 Bermejo I, Holzel LP, Kriston L et al. Subjektiv erlebte Barrieren von Personen mit Migrationshintergrund bei der Inanspruchnahme von Gesundheitsmaßnahmen. Bundesgesundheitsblatt Gesundheitsforschung Gesundheitsschutz 2012; 55: 944-953

5 Blum K, Steffen P. Kultursensibilität der Krankenhäuser in Nordrhein-Westfalen. In. Düsseldorf: Deutsches Krankenhausinstitut e. V.; 2012: 1-100

6 Borde T, David M, Kentenich H. Erwartungen und Zufriedenheit deutscher und türkischsprachiger Patientinnen im Krankenhaus - eine vergleichende Befragung in einer Berliner Frauenklinik. Gesundheitswesen 2002; 64: 476-485

\section{Konsequenz für Klinik und Praxis}

- Patienten mit türkischem Migrationshintergrund $(T)$ ist ihre Religion mehrheitlich „ziemlich“ bis „äußerst“ wichtig. Bei ihrer stationären Betreuung sollte daher religiösen Aspekten eine hohe Priorität beigemessen werden.

- Da die Mehrzahl der T die Möglichkeit zum Gebet als "schlecht“ oder „sehr schlecht“ wahrnimmt, sollte die Einrichtung eines muslimischen Gebetsraumes in Krankenhäusern mit einem relevanten Anteil an T unbedingt erwogen werden. Auch die Zusammenarbeit mit islamischen Geistlichen sollte angestrebt werden.

- Unsere Studie zeigt, dass mehr als einem Drittel der T die Einhaltung islamischer Ernährungsgebote „ziemlich“ oder „äußerst“ wichtig ist. 90\% der befragten T halten es jedoch für „ziemlich“ oder „äußerst“ schwierig, diese Gebote im Krankenhaus einzuhalten. Eine Kennzeichnung der Nahrungsmittel, am besten auf Türkisch, oder speziell abgestimmte Speisepläne könnten die Situation ohne großen Mehraufwand deutlich verbessern.

- In unserer Untersuchung wünscht sich erwartungsgemäß die Mehrheit der weiblichen T eine Betreuung durch gleichgeschlechtliche Pflegekräfte oder Ärztinnen. Neu ist jedoch, dass auch männlichen T eine gleichgeschlechtliche pflegerische und ärztliche Betreuung wichtiger ist als deutschen Männern und Frauen. Korrespondierend dazu wünschen sich T eher als D die Anwesenheit einer gleichgeschlechtlichen dritten Person bei Untersuchungen oder Behandlungen. Da insbesondere die Krankenpflege überwiegend weiblich ist, dürfte es für viele Krankenhäuser eine Herausforderung darstellen, sich auf den Wunsch eines Teils der Männer mit türkischem Migrationshintergrund nach einer gleichgeschlechtlichen Betreuung einzustellen.

7 Duschek K, Weinmann J, Boehm K et al. Leben in Deutschland Haushalte, Familien und Gesundheit - Ergebnisse des Mikrozensus 2005. In: Bundesamt S ed. Wiesbaden: Statistisches Bundesamt; 2006:1-95

8 Giese A, Uyar M, Uslucan $\mathrm{H}$ et al. How do hospitalised patients with Turkish migration background estimate their language skills and their comprehension of medical information - a prospective cross-sectional study and comparison to native patients in Germany to assess the language barrier and the need for translation. BMC Health Services Research 2013; 13: 196

9 Ilkilic I. Begegnung und Umgang mit muslimischen Patienten. Eine Handreichung für die Gesundheitsberufe. Bochum: Zentrum für Medizinische Ethik; 2006

10 Knipper M, Bilgin Y. Migration und Gesundheit. St. Augustin/Berlin: Konrad-Adenauer-Stiftung e. V., Sankt Augustin / Berlin: Türkisch-Deutsche Gesundheitsstiftung e.V. 2009

11 Lenth R. Some Practical Guidelines for Effective Sample Size Determination. Am Stat 2001; 55: 187-193 
12 Liljeberg H. Deutsch-Türkische Lebens- und Wertewelten 2012. In. Berlin: INFO GmbH und LILJEBERG Research International Ltd. Sti.; 2012

13 Razum O, Geiger I, Zeeb H, et al. Gesundheitsversorgung von Migranten. Dtsch Arztebl 2004; 101 : 2882-2887

14 Salman R, Weyers S. MiMi Project - With Migrants for Migrants. In: Koller T, Hrsg. Poverty and social exclusion in the WHO European Region: health systems respond. Copenhagen, Denmark: WHO Regional Office for Europe; 2010:52-63

15 Sauer M. Ergebnisse der zwölften Mehrthemenbefragung 2011. In, Integrationsprozesse türkeistämmiger Migrantinnen und Migranten in Nordrhein-Westfalen Essen, Germany: Stiftung Zentrum für Türkeistudien und Integrationsforschung; 2012:1-228
16 Sauer M, Halm D. Methodik und Durchführung der Befragung. In: Sauer M, Halm D, Hrsg. Erfolge und Defizite der Integration türkeistämmiger Einwanderer Entwicklung der Lebenssituation 1999 bis 2008. Wiesbaden: VS Verlag für Sozialwissenschaften; 2009:132-133

17 Tagay S, Dullmann S, Brahler E et al. Wie hängen Religiosität, psychische Symptombelastung und Integration zusammen? Eine vergleichende Betrachtung der Religiosität bei türkischen / kurdischen Migranten gegenüber Deutschen. Psychiat Prax 2009; 36: $286-292$

18 Yildirim-Fahlbusch Y. Türkische Migranten: Kulturelle Missverständnisse. Dtsch Arztebl 2003; 100: A-1179
Interessenkonflikt

Die Autoren erklären, dass keine finanziellen Konflikte bestehen.

DOI 10.1055/s-0041-100007 Dtsch Med Wochenschr 2015: 140: e14-e20

(c) Georg Thieme Verlag KG . Stuttgart - New York .

ISSN 0012-0472 